\title{
An indirect tire identification method based on a two-layered fuzzy scheme
}

\author{
Dailin Zhang*, Dengming Zhang, Jingming Xie and Youping Chen \\ School of Mechanical Science and Engineering, Huazhong University of Science and Technology, Hubei, China
}

\begin{abstract}
While the heights and numbers of tires in the assembly line are changing, the traditional methods are difficult to identify the types of the tires, so an indirect tire identification method is proposed to identify the types of the tires, which uses the widths of the tires, the diameters and the shapes of the hubs to classify the tires. First, the tires are identified according to the widths and diameters by using the fuzzy C-means clustering algorithm. Second, the shapes of the hubs are used to distinguish the tires with similar dimensions. Finally, a two-layered fuzzy scheme is designed to identify the tires. Experimental results show that the two-layered fuzzy scheme is more effective than the fuzzy C-means clustering algorithm. And the proposed indirect tire identification method can achieve an accuracy of above $99.9 \%$ in the assembly line of tires.
\end{abstract}

Keywords: Fuzzy C-means clustering algorithm, two-layered fuzzy scheme, tire identification, machine vision

\section{Introduction}

Generally, an automobile tire is identified by a barcode molding on the sidewall, from which we can get almost all parameters of the tire necessary for automobile plants. The barcode can be scanned by a machine vision system and identified by the obtained characters $[1,6]$. The identified parameters of a tire are used for the tracing task in an automobile production line. But if several tires are overlapped together and sent to an assembly station of an automobile assembly line, it is very difficult to accurately recognize the characters by using a camera because various heights with different groups of tires will influence on the image quality. So the paper proposes an indirection tire identification method based on a two-layered fuzzy scheme.

Nowadays, there are two main clustering strategies: the hard clustering scheme and the fuzzy clustering scheme. K-means clustering algorithm is a generally

\footnotetext{
${ }^{*}$ Corresponding author. Dailin Zhang, School of Mechanical Science and Engineering, Huazhong University of Science and Technology, Hubei, China. Tel./Fax: +86 2787543555; E-mail: mnizhang@mail.hust.edu.cn.
}

used hard clustering scheme and the fuzzy C-means clustering algorithm is a generally used fuzzy clustering scheme. Compared with the hard clustering methods, the fuzzy clustering algorithm described by a membership function can avoid some unfavorable factors, such as the poor contrast, overlapping intensities, noises and intensity in homogeneities. So the fuzzy clustering algorithm achieved many applications in many areas. For example, in [4] a generalization method of the fuzzy local information C-means clustering algorithm was proposed in order to be applicable to any kind of input data sets instead of images. Mansoori proposed a novel fuzzy rule-based clustering algorithm (FRBC), which could employ a supervised classification approach to do the unsupervised cluster analysis [3]. Nefti-Meziani, et al. improved fuzzy clustering algorithm by using an inclusion concept that allows the determination of the class prototype which covers all the patterns of that class [5].

Based on the measured dimensions of the tires, such as the widths of the tires and the diameters of the hubs, the fuzzy C-means clustering algorithm can be used to identify the tires. But the fuzzy C-means 
clustering algorithm has some disadvantages because of its self-structure [5, 7]. One of the disadvantages in the identification of the tires is that some types of tires with similar dimensions cannot be identified accurately by using the fuzzy C-means clustering algorithm. In order to identify all types of tires, a two-layered fuzzy scheme is proposed, which includes two layers: Level 0 and Level 1. In Level 0, the tires with similar dimensions are considered as one class and the tires are identified by the fuzzy C-means clustering algorithm, and in Level 1 the tires with similar dimensions are identified by fuzzy interference rules.

\section{Indirect tire identification method based on a two-layered fuzzy scheme}

Instead of scanning a barcode, the paper uses the tire dimension parameters measured by a machine vision system to identify tires. The measured tire dimension parameters are the widths of the tires and diameters of the hubs. According to the dimension parameters, the fuzzy C-means clustering algorithm is used to identify the tire types. But some tires with similar dimensions cannot be identified by the fuzzy C-means clustering algorithm, so a two-layered fuzzy scheme is proposed to identify the tires.

\subsection{Measurement of the tire dimension parameters}

As shown in Fig. 1, the tire identification system includes a width measurement system, a diameter measurement system and a template matching system. When the tires are being transferred in the assembly line, a group of tires will have an identical type which can be identified by the designed indirect tire identification system. According to the identification results, the tires are transferred to different branches to assembly the different types of cars. The width measurement system mainly includes two cameras beside the assembly line and the width and number of the tires are calculated from the images taken by the two cameras. The diameter measurement and template matching system mainly include a camera equipped on the top of the assembly line, by which the diameter of the top hub is measured and the shape of the top hub is matched.

From the width measurement system the height of the overlapped tires can be obtained by the side cameras, which is the total height of the tires. A group of overlapped tires have an identical width $w$, which can be calculated by

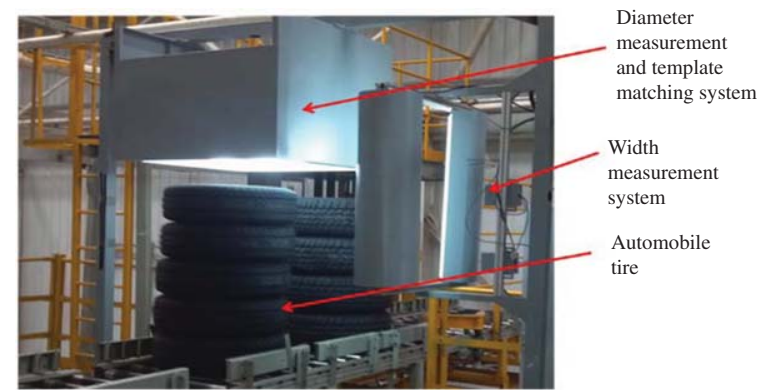

Fig. 1. Indirect tire identification system of tires.

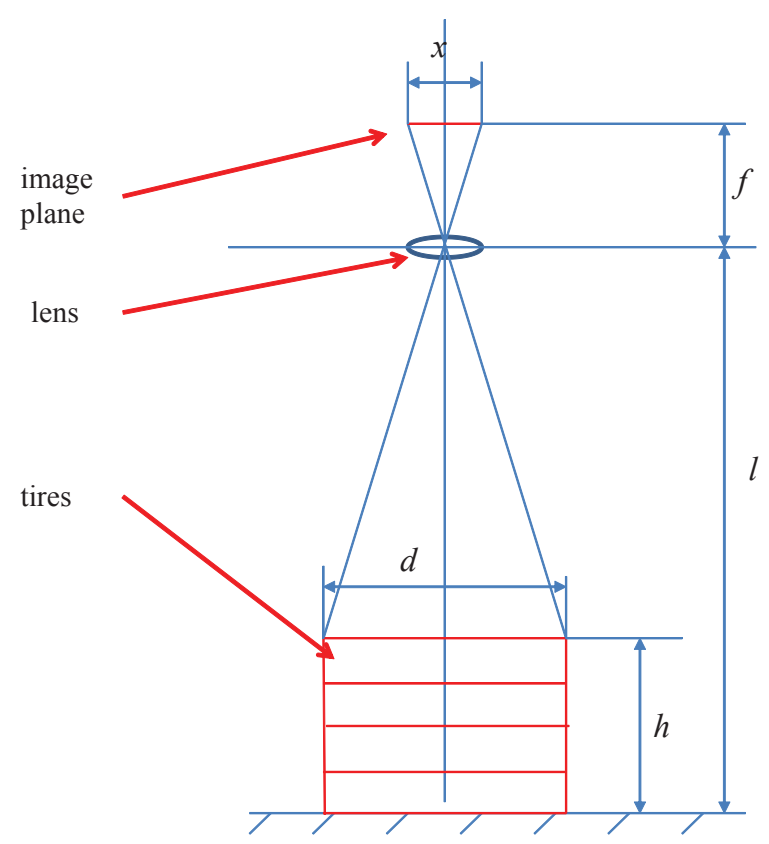

Fig. 2. Diameter measurement schematic diagram by the pinhole camera model.

$$
w=\frac{h}{n}
$$

Where $h$ and $n$ are the height and the number of the overlapped tires, respectively.

The pixels of the top hub can be calculated by the image taken by the top camera. Combined with the height $h$, the diameter of the top hub can be calculated by the pinhole camera model shown in Fig. 2. In Fig. 2, $f$ is the focus length, $x$ and $d$ are the physical dimension in the camera and the diameter of the hub, respectively. $l$ is the distance from the lenses to the ground. 
According to the pinhole camera model, the following equation can be obtained

$$
\frac{x}{d}=\frac{f}{l-h}
$$

Then the diameter of the top hub can be calculated by

$$
d=\frac{l-h}{f} x
$$

If the two above dimensions are calculated accurately, the tires can be identified. But because of the varying intensity of light, the different positions of the tires and others factors, the measurement accuracies of the two dimensions are not so high that the measured dimensions of tires have big errors.

A fuzzy C-means clustering algorithm can overcome the inaccuracies of the dimensions to some degrees. But if the difference between the dimensions of two groups of tires is very small, the fuzzy C-means clustering algorithm cannot identify the tires accurately. According to our analysis, the shapes of the hubs are different if the dimensions are similar. So the template matching method can be used to generate the third feature of the tires, i.e, the similarities between a hub and the matched hub templates.

By using the shape-based matching method in [2], a score $s(s \in[0,1])$ can be achieved by measuring the similarity between a hub and a matched hub template. The score can be considered as the degree of membership of the hub in the matched hub template.

\subsection{Identification method based on the fuzzy C-means clustering algorithm}

The fuzzy C-means clustering algorithm is used to identify the tires according to the measured dimensions. Before the identification process the tires with similar dimensions are considered as one class, and the number of clusters is known. Then the fuzzy C-means clustering algorithm can be used to design a supervised identification system and the procedures can be described as follows.

First, the dimensions of all types of the sample tires are used to obtain the optimum parameters for the fuzzy $\mathrm{C}$-means clustering algorithm, including the optimum membership function matrix $U$ and the optimum centers of the clusters. And the optimum parameters are stored in the computer.

Second, new tires are identified by using the optimized fuzzy C-means clustering algorithm. In the process, the dimensions of a new tire are considered as a new sample and added to the old sample sets.

Third, obtain the maximum degree of membership. Finally, the clustering class index corresponding to the maximum degree of membership indicates the identified type.

In the fuzzy C-means clustering algorithm, the following objective function is applied

$$
J_{m}=\sum_{j=1}^{N} \sum_{i=1}^{m}\left[\mu_{j}\left(x_{i}\right)\right]^{b}\left\|x_{i}-c_{j}\right\|^{2}
$$

Where $b$ is a real number greater than 1 , and generally it is be set for 2. $m$ represents the number of the sample data which is any real number greater than 1. $x_{i}$ is the $i$ th sample data and $i=1,2, \ldots, m . N$ is the number of the clusters. $\|*\|^{2}$ is any norm expressing the similarity between any sample data and the cluster center. by

$c_{j}$ is the $j$ th cluster center, which can be calculated

$$
c_{j}=\frac{\sum_{k=1}^{m}\left[\mu_{j}\left(x_{i}\right)\right]^{b} x_{i}}{\sum_{k=1}^{m}\left[\mu_{j}\left(x_{i}\right)\right]^{b}}
$$

$\mu_{j}\left(x_{i}\right)$ is the degree of membership of $x_{i}$ in the $j$ th cluster, which can be calculated by

$$
\mu_{j}\left(x_{i}\right)=\frac{1}{\sum_{k=1}^{N}\left(\frac{\left\|x_{i}-c_{j}\right\|}{\left\|x_{i}-c_{k}\right\|}\right)^{\frac{2}{b-1}}}
$$

And it meets the following condition

$$
\sum_{i=1}^{m} \mu_{j}\left(x_{i}\right)=1
$$

Fuzzy classification is carried out through an iterative optimization of the objective function shown in Equation (4), with the update of membership $\mu_{j}\left(x_{i}\right)$ and the cluster centers $c_{j}$. The iteration will stop when max $\left\{\left\|\mu_{i j}^{(k+1)}-m u_{i j}^{(k)}\right\|\right\}<\epsilon$, where $\mu_{i j}=$ $\mu_{i}\left(x_{j}\right)$ and $\epsilon$ is a termination criterion between 0 and 1 and $k$ is the iteration step number. This procedure converges to a local minimum or a saddle point of $J_{m}$.

The iterative optimization of the objective function can be done by the following steps.

Step 1. set the number of clusters to be the type number of the tires $N$ and set $b$ to be 2;

Step 2. initialize $U=\left[\mu_{i j}\right]$ matrix as $U^{(0)}$, where $\mu_{i j}=\mu_{i}\left(x_{j}\right)$ and $U^{(0)}$ is any $N \times m$ matrix; 
Step 3. update the cluster center $c_{j}$ according to Equation (5);

Step 4. update $U^{(k)}$ and $U^{(k+1)}$. The updated cluster centers are used to update the degrees of membership according to Equation (6) and the updated degree of membership is $\mu_{i j}^{(k+1)}$, where $k=0,1,2, \ldots$ denotes the iteration times;

Step 5. calculate $\max \left\{\left\|U^{(k+1)}-U^{(k)}\right\|\right\}$.

If $\max \left\{\left\|U^{(k+1)}-U^{(k)}\right\|\right\}<\varepsilon$, then stop the iteration; otherwise, repeat step 3-step 5.

Finally, an optimum membership function matrix $U=\left[\mu_{i j}\right]$ is obtained from the sample tires, which can be used to identify the other tires in the assembly lines of tires.

After the optimum membership function matrix and the optimum centers of the clusters are obtained, the following three steps are used to identify the tires on line.

Step 6. if $x_{m+1}$ is the new measured data, set $j=$ $1,2, \ldots, N$ and calculate the degree of membership of $x_{m+1}$ in the $j$ th cluster

$$
\mu_{j}\left(x_{i}\right)=\frac{1}{\sum_{k=1}^{N}\left(\frac{\left\|x_{m+1}-c_{j}\right\|}{\left\|x_{m+1}-c_{k}\right\|}\right)^{\frac{2}{b-1}}}
$$

Step 7. search the maximum degree of membership $\max U$ for $j=1,2, \ldots, N$ by

$$
\left.\max U\right|_{j=i n d e x} U=\max \left\{\mu_{j}\left(x_{i}\right)\right\}
$$

where index $U$ is the corresponding cluster index when the maximum degree of membership $\max U$ is obtained

Step 8. obtain the corresponding cluster index index $U$ when the maximum degree of membership $\max U$ is obtained. Then the class corresponding to the clustering index index $U$ is the identified tire type.

By using the steps 6-8, every tire in the assembly lines of tires can be identified on line.

\subsection{Identification method of the two-layered fuzzy scheme}

The tires with similar dimensions may generate an identical degree of membership $\mu_{i j}$, so they cannot be identified by the fuzzy $\mathrm{C}$-means clustering algorithm in Section 2.2. In order to identify the tires with similar dimensions, a two-layered fuzzy scheme shown in Fig. 3 is proposed.

In the two-layered fuzzy scheme, the clusters with similar dimensions are considered as one cluster, which is identified by the fuzzy C-means clustering algorithm

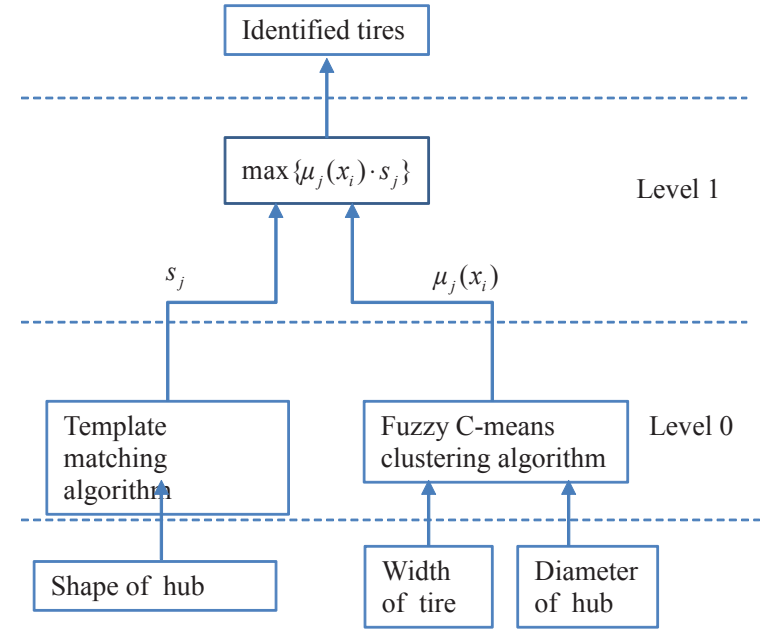

Fig. 3. Schematic diagram of the two-layered fuzzy scheme.

in Level 0. Combined with the identified results, the template matching algorithm is used to generate the similarities and the fuzzy inference rules are used to identify the tires with similar dimensions in Level 1.

As shown in Fig. 3, the fuzzy C-means clustering algorithm is used to identify the tires in Level 0 and the degree of membership $\mu_{i j}$ can be obtained. Then the template matching algorithm is used to obtain the similarities between a hub and the matching hub templates by using the shape-based matching method. The similarity $s_{j}\left(s_{j} \in[0,1]\right)$ can be considered as the degree of membership in the $j$ th hub template. Then the fuzzy inference is used to obtain the type of every tire in Level 1.

Because the template matching method is time-cost work, it is only used to distinguish the tires which cannot be identified by the fuzzy C-means clustering algorithm.

It is supposed that $u$ clusters need to be further identified after executing the fuzzy $\mathrm{C}$-means clustering algorithm. If the $u$ clusters include $w$ types of tires, the following fuzzy rules can be used to calculate the fuzzy degrees of membership $\mu_{j}^{\prime}\left(x_{i}\right)$

$$
\mu_{j}^{\prime}\left(x_{i}\right)=\mu_{k}\left(x_{i}\right) \cdot s_{j}
$$

Where $k=1,2, \ldots, w$ and $j=1,2, \ldots, w \cdot x_{i}$ indicates the $i$ th new sample data and $\mu_{j}^{\prime}\left(x_{i}\right)$ is the new degree of membership of $x_{i}$ in the $j$ th cluster.

By Equation (10) the degrees of membership of $w$ clusters are calculated corresponding to $w$ types of tires. Then the tires can be identified by searching the maximum degree of membership by 


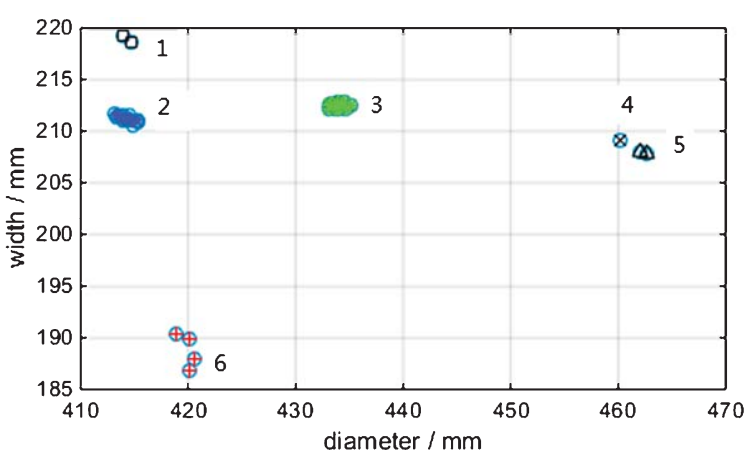

Fig. 4. Experimental results by the fuzzy C-means clustering algorithm.

$$
\left.\max U^{\prime}\right|_{j=i n d e x U^{\prime}}=\max \left\{\mu_{j}^{\prime}\left(x_{i}\right)\right\}
$$

The index index $U^{\prime}$ is the corresponding cluster index when the maximum degree of membership is obtained. And finally, the class corresponding to the clustering index index $U^{\prime}$ is the identified tire type.

It is noticed that the two-layered fuzzy scheme can be applied to the other cluster analysis of similar objects. In order to partition the similar objects, they are considered as one class and partitioned by the basic fuzzy C-means clustering algorithm in Level 0 . Then other features are combined together to finish the final partition in Level 1.

\section{Experimental results}

The experiments are made to verify the effectiveness of the two-layered fuzzy scheme.

36 measured tire data are set as the sample data and there are 6 clusters according to our statistics. In the fuzzy C-means clustering algorithm, $b$ is set to be 2 . The identified result by the fuzzy C-means clustering algorithm is shown in Fig. 4, from which it can be seen that the tires are classified into 6 clusters, but the result is incorrect. In fact, Class 4 and Class 5 are an identical type, Class 6 includes two types.

The experimental results by the two-layered fuzzy scheme are shown in Fig. 5. The results are presented by two steps. First, Fig. 5(a) shows that the fuzzy C-means clustering algorithm classifies sample data into 5 classes and Class 5 includes two types of tires with similar dimensions. Second, the fuzzy scheme classifies Class 5 into two classes accurately. And finally, the sample data are classified into 6 classes accurately.

From Fig. 5, it can be concluded the sample data are correctly classified. In the assembly line of automobile tires shown in Fig. 1, the identification accuracy of the tires can reach up to above $99.9 \%$.

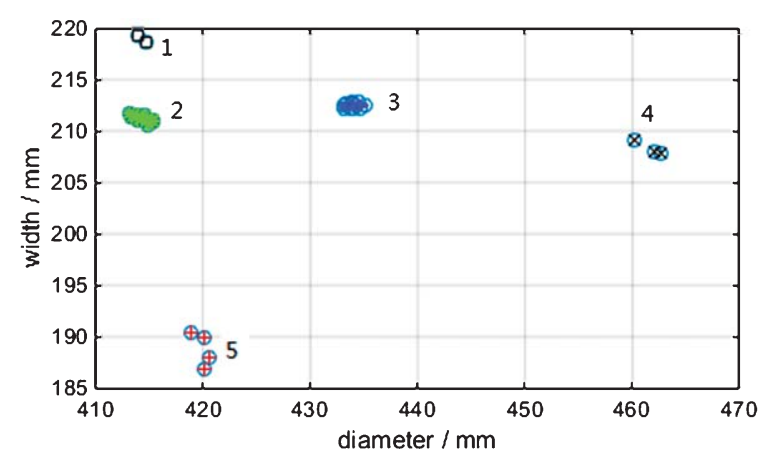

(a) 5 clusters in Level 0

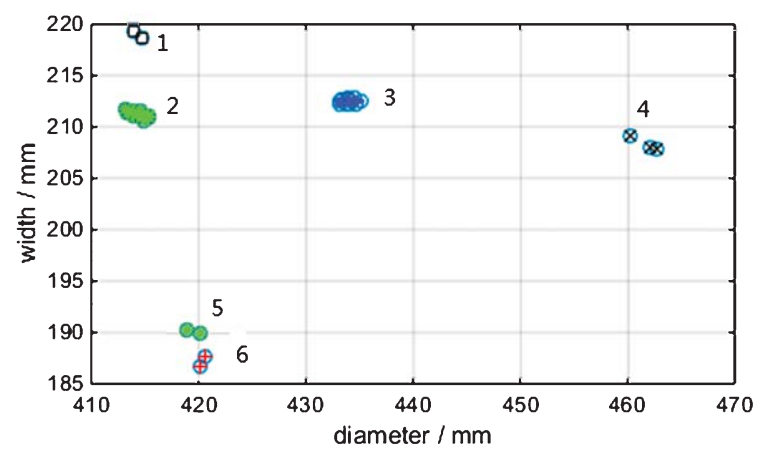

(b) 6 clusters in Level 1

Fig. 5. Experimental results by the two-layered fuzzy scheme.

In the paper, there is only one cluster which needs to be further classified. But there would be more similar clusters because of more types of tires in the future.

\section{Conclusions}

The paper proposed an indirect tire identification method based on a two-layered fuzzy scheme. Through the three cameras the widths of the tires, the diameters and shapes of the hubs are obtained. By using the measured dimension data, a fuzzy C-means clustering algorithm is designed to identify the tires. But some types of tires with similar dimensions cannot be accurately identified by the fuzzy $\mathrm{C}$-means clustering algorithm. In order to identify all tires accurately, a twolayered fuzzy scheme is finally designed to identify the tires with similar dimensions.

Experimental results show that the two-layered fuzzy scheme can identify all the types of tires and achieve higher identification accuracy than the fuzzy C-means clustering algorithm. And the developed indirect tire 
identification system is applied to the assembly line of tires and achieves an identification accuracy of above $99.9 \%$.

\section{References}

[1] Cognex, DataMan barcode readers guide, 2015.

[2] C. Steger, Occlusion, clutter, and illumination invariant object recognition, International Archives of Photogrammetry and Remote Sensing 34 (2002), 345-350.

[3] E.G. Mansoori, FRBC: A fuzzy rule-based clustering algorithm, IEEE Transactions on Fuzzy Systems 19 (2011), 960-971.
[4] S. Krinidis and M. Krinidis, Generalised fuzzy local information C-means clustering algorithm, Electronics Letters 48 (2012), $1468-1470$.

[5] S. Nefti-Meziani, M. Oussalah and M. Soufian, On the use of inclusion structure in fuzzy clustering algorithm in case of Gaussian membership functions, Journal of Intelligent \& Fuzzy Systems 28 (2015), 1477-1493.

[6] X. Yu, P. Gou and L. Su, Study on tire sidewall marking recognition based on moments, ICINIS2013, Shenyang, China, 2013, pp. 21-24.

[7] Y.H. Zheng, B. Jeon, D.H. Xu, Q.M. Jonathan Wu and H. Zhang, Image segmentation by generalized hierarchical fuzzy C-means algorithm, Journal of Intelligent \& Fuzzy Systems 28 (2015), 961-973. 